\title{
A monetary incentive increases postal survey response rates for pharmacists
}

\author{
Christine L Paul, Raoul A Walsh, Flora Tzelepis
}

J Epidemiol Community Health 2005;59:1099-1101. doi: 10.1136/jech.2005.037143

Background: Achieving acceptable response rates from health care providers via postal questionnaires is an ongoing challenge. The use of monetary incentives is one of the most effective strategies for increasing response rates. However, the effect and cost of such an incentive on retail pharmacists' response rates has not been well studied.

Methods: A sample of 700 pharmacies was selected at random from the electronic Yellow Pages in NSW Australia and mailed a brief survey regarding pharmacotherapies and advice for smoking cessation. Half of the sample was randomly allocated to receive an offer of an US\$14 gift voucher.

Results: The response rates were $65.9 \%$ for the voucher group and $53.5 \%$ for the no-voucher group. The odds of response from the voucher group was $1.68(95 \% \mathrm{Cl}=1.23$, $2.30)$ times greater than for the no-voucher group. The cost per additional respondent was US\$67.95. The incentive also reduced follow up costs by $10 \%$.

Conclusions: A moderately sized monetary incentive is able to achieve a significant increase in response rates for retail pharmacists, thereby reducing potential bias in the sample.

$\mathrm{P}$ ostal surveys are a well used tool for collecting data for a range of health related settings. ${ }^{1-3}$ Achieving acceptable response rates is crucial to minimising bias $^{4}$ and remains a challenge, particularly for studies of health care providers. ${ }^{35}$ A number of strategies have been trialled to maximise response rates, with one of the most effective strategies being the use of a monetary incentive. ${ }^{6}$ While monetary incentives can increase the odds of response by a factor of 2.02, the effect is dependent on factors such as the amount of the incentive. $^{6-8}$

The potential importance of population type has been acknowledged, yet most of the research into the effect of monetary incentives for health care providers has focused on medical practitioners. ${ }^{6}$ Other important community based health care providers such as retail or community pharmacists have received little attention, despite this group's potential to modify population health risk behaviours because of their contact with large numbers of people. This group of health care providers practise in a highly commercialised environment with frequent contact from pharmaceutical companies that can offer substantial incentives for participation in studies. Given these factors and the proliferation of the use of incentives in all forms of marketing, it is important to continue to study the effects of small to medium incentives on response rates, particularly for little studied groups such as pharmacists.

This study aimed to explore the impact of a US\$14 incentive on response rates for pharmacists in NSW, Australia.

\section{METHOD}

A sample of 700 pharmacies was selected at random from the electronic Yellow Pages for NSW, Australia. Pharmacies were eligible to participate if they had sold any nicotine replacement therapy (NRT) or bupropion in the previous month. Eligibility was self assessed by the pharmacist completing a screening item on the front of the survey. The accuracy of recall was expected to decrease over time, therefore a period of one month was selected as the maximum time since contact with a customer that would provide acceptable recall. The pharmacist in charge in each selected pharmacy was mailed a primer postcard followed by the questionnaire within the following two weeks. Non-responders received one print reminder four weeks after receipt of the questionnaire. Telephone reminders were provided a further two to six weeks later.

Half of the sample was randomly allocated using random number generation to receive an offer of a AU\$20 (US\$14) gift voucher (voucher group) as partial reimbursement for the costs incurred in completing the questionnaire. The vouchers could be redeemed at any one of a number of large supermarkets and department stores throughout Australia. For the voucher group, both the questionnaire and the accompanying letter stated that the voucher would be mailed to the participant once the completed questionnaire had been received. The authors were aware of participant's group allocation but had no direct contact with the participants during the mail out or follow up process.

The study was approved by the University of Newcastle Human Research Ethics Committee with the proviso that after data collection was completed, all participants be advised that only half of the sample had been offered the voucher and be given the opportunity to withdraw their data.

The questionnaire took about 10 minutes to complete and was six pages in length with items well spaced and in large font. The 28 items included items relating to the provision of advice to NRT and Zyban purchasers, attitudes toward supporting smoking cessation in the pharmacy environment, and training received regarding NRT and smoking cessation. The content of the data collected from the pharmacists is reported elsewhere.

\section{RESULTS}

Of the 700 pharmacies selected in the sample, 35 were found to be ineligible (returned survey showed no NRT or Zyban sold in past month, pharmacy closed, wrong address, duplicate, or no pharmacist at the pharmacy). Of the remaining 665 pharmacies, 397 returned a completed questionnaire (response rate $=59.7 \%$ ).

For the voucher group, 220 of the 334 eligible pharmacists returned a completed questionnaire, giving a response rate of $65.9 \%$. For the no-voucher group, 177 of the 331 eligible pharmacists returned a questionnaire, giving a response rate of $53.5 \%$. The difference between these two proportions was significant $\left(\chi^{2}=10.6, \quad \mathrm{df}=1, \quad \mathrm{p}<0.005 \quad \mathrm{OR}=1.68\right.$, 


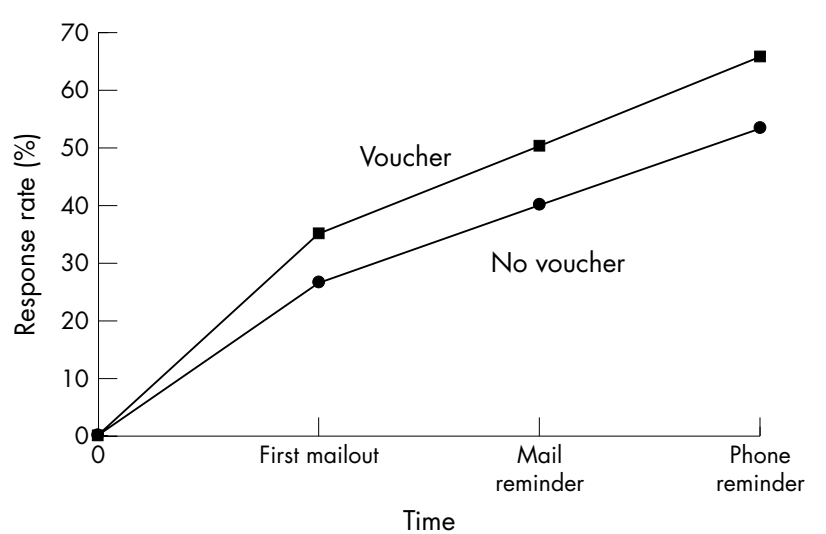

Figure 1 Effect of the questionnaire mail out, print reminder, and telephone reminder on response rates.

$95 \% \mathrm{CI}=1.23,2.30)$. Of the 220 voucher group pharmacists who returned a questionnaire, $21(9.5 \%)$ indicated they did not want to receive the voucher.

After the mailing of a letter disclosing that the study involved two groups, only one of which received a voucher, 11 participants withdrew from each group. This gave final response rates of $62.6 \%$ for the voucher group and $50.2 \%$ for the no-voucher group. The difference between these two proportions was significant $\left(\chi^{2}=10.4, \mathrm{df}=1, \mathrm{p}<0.005\right.$; $\mathrm{OR}=1.66,95 \% \mathrm{CI}=1.22,2.26)$. Figure 1 shows the effect of the first mail out, print reminder, and telephone reminder on response rates.

The cost per additional respondent of the vouchers was calculated as follows:

$$
\begin{array}{ll}
\begin{array}{ll}
\text { Cost per additional } \\
\text { respondent }
\end{array} & \begin{array}{l}
\text { Number of completed voucher } \\
\text { group surveys } \times \text { US } \$ 14 \\
\text { in voucher group }
\end{array} \\
& =\frac{220 \times \text { US } \$ 14}{41}
\end{array}
$$

Therefore, the cost per additional respondent was US $\$ 75.12$. If the number of pharmacists $(n=21)$ who did not want to be sent the voucher is deducted from the numerator, the cost per additional respondent is US\$67.95. If the cost of mailing the voucher to those who complete the survey was included in the calculations, the cost per additional respondent would be slightly higher. The use of the voucher also decreased follow up costs by about $10 \%$ at each follow up point, based on the additional printing, mailing, and telephone costs that would have been incurred had the voucher group response rates been the same as the no voucher group response rates.

\section{DISCUSSION AND CONCLUSION}

The use of a moderate monetary incentive produced a significant increase in the number of pharmacists who completed a health related questionnaire. An increase in response rates from $54 \%$ to $66 \%$ is both statistically significant and probably reduces the potential for bias in the sample. The increased odds of a response, at 1.68 is slightly lower than the 2.02 found by Edwards' et al systematic review. ${ }^{6}$ Given that previous research has found higher response rates are likely to be achieved by a nonconditional incentive, rather than the conditional incentive used in this study, double the odds of a response as found by Edwards et $a l^{6}$ may be achievable with this population. When considered in terms of the cost of US\$67.95 per additional

\section{What this paper adds}

What is already known on this subject?

A number of studies have shown that health care providers such as medical doctors are more likely to respond to health surveys if a monetary incentive is provided. Little is known about the influence of incentives on response rates for other important health care provider groups such as retail or community pharmacists.

What does this study add?

This study shows that a significant increase in pharmacists' response rates to a postal survey can be achieved using a modest monetary incentive, and that this incentive acts independently from using reminders that also increase response rates.

respondent, smaller incentives are worth exploring with this population. The generalisability of the finding outside the Australian context must be considered. However, given that the finding is not dissimilar to other studies of incentives ${ }^{6}$ the result may be generalisable.

\section{Policy implications}

The use of incentives to increase response rates imposes an additional cost on research funds, yet seems to significantly increase response rates, even for health care providers in a highly commercialised environment. Therefore, researchers even on limited funds need to consider the potential benefits of incentives as a matter of course.

It should be noted that the study included the use of a primer postcard and reminders, each of which has a significant effect on response rates. A factorial design that would have isolated the effect of the incentive, was not possible in this trial given the level of funding available. However, figure 1 shows that the impact of the incentive was to increase the response to the initial mail out. The two reminders seemed to have a similar impact on both study groups, independent from the effect of the voucher.

Clearly, a useful increase in the response rates of retail pharmacists is achievable with a moderately sized monetary incentive, although the incremental cost may be considered high.

\section{ACKNOWLEDGEMENTS}

The work of Richard Turner and Deanna Wolfgang on this project is acknowledged.

\section{Authors' affiliations}

C L Paul, R A Walsh, F Tzelepis, Centre for Health Research and Psychooncology (CHeRP), Wallsend, NSW Australia

Funding: this paper was prepared by the Centre for Health Research and Psycho-oncology (CHeRP). The project was funded by the University of Newcastle Research Grants Committee, the Cancer Council NSW, and the Hunter Medical Research Institute. The views expressed are not necessarily those of the Cancer Council NSW.

Competing interests: none declared.

Correspondence to: Dr C Paul, Centre for Health Research and Psychooncology (CHeRP), Locked Bag No 10, Wallsend 2287, NSW,

Australia; Chris.Paul@newcastle.edu.au

Accepted for publication 20 July 2005 


\section{REFERENCES}

1 Parker CJ, Dewey ME. Assessing research outcomes by postal questionnaire with telephone follow-up. Int J Epidemiol 2000;29:1065-9.

2 Harrison RA, Holt D, Elton PJ. Do postage stamps increase response rates to postal surveys? A randomised controlled trial. Int J Epidemiol 2002;31:872-4.

3 Moses SH, Clark TJ. Effect of a prize draw incentive on the response rate to a postal survey of obstetricians and gynaecologists: a randomised controlled trial. BMC Health Services Research 2004;4. http//www.biomedcentral.com/ 1472-6963/4/14.

4 Armstrong BK, White E, Saracci R. Principles of exposure measurement in epidemiology. Monographs in epidemiology and biostatistics. Vol 21. New York: Oxford University Press, 1995:294-321.
5 McAvoy BR, Kaner EF. General practice surveys: a questionnaire too far? BMJ 1996;313:732-3

6 Edwards P, Roberts I, Clarke $M$, et al. Increasing response rates to postal questionnaires: systematic review. BMJ 2002;324:1183.

7 Halpern S, Ubel P, Berlin JA, et al. Randomised trial of $\$ 5$ versus $\$ 10$ monetary incentives, envelope size, and candy to increase physician response rates to mailed questionnaires. Med Care 2002;40:834-9.

8 Kasprzyk D, Montano DE, StLawrence JS, et al. The effects of variations in mode of delivery and monetary incentives on physicians' responses to a mailed survey assessing STD practice patterns. Evaluation and the Health Professions $2001 ; 24: 3-17$

\section{THE JECH GALLERY}

\section{Visual impairment and new technologies}

7 he term "visual impairment" encompasses a variety of conditions. A range of equipment is available to enable people with sight loss to participate in various activities. Some of these have been around for a long time: the first book in braille was published in 1827; guide dogs for the blind were first formally trained a century later. New technologies-such as computer software-are constantly being developed and what is now available means that there is no reason why a blind person cannot be employed in a job that is primarily computer based.

These pictures show Mark Kirkham, who is totally blind, working for the South West Public Health Observatory on a project analysing data on road traffic collisions. Jaws screenreader software has been installed on Mark's PC, allowing him to use many other applications including Microsoft Access and Excel. Jaws software, manufactured by Freedom Scientific, reads aloud all the text information that would normally appear on the screen of a sighted person. Jaws may be configured in a variety of ways so that different types of information are spoken appropriately. For example, it will read at different speeds and can be set to read either all changing information on the screen or only what is currently highlighted. Where a sighted person would often use a mouse, Jaws makes use of keyboard shortcut commands to perform functions such as navigating through dialogue boxes and activating buttons.

The image at the top shows Mark reading braille, the bottom right image shows him wearing headphones enabling him to listen to Jaws without disturbing colleagues in a busy office. The third image (bottom left) illustrates Mark using a "Braille 'n Speak", a stand alone note taking device. It uses a braille keyboard and, like Jaws, has a voice output. It is portable, which means it is suitable for Mark to quietly take notes in meetings. The machine also has a serial port, allowing it to communicate with mainstream devices such as printers and PCs. The equipment needed by any person with a visual impairment will reflect their personal needs, circumstances, and preferences. It has the potential to bring about inclusion in terms of both social and employment opportunities.
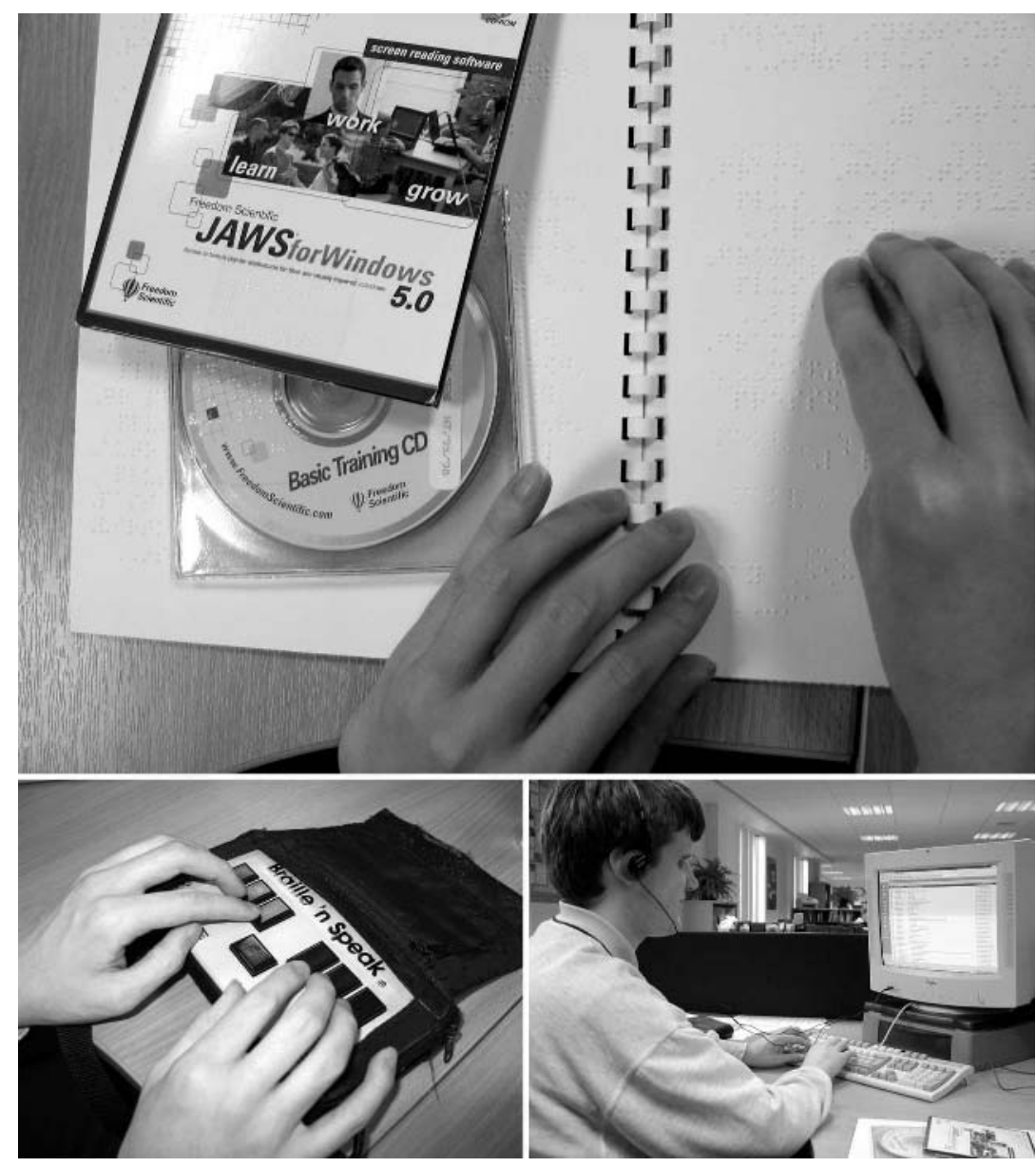

Mary E Shaw

South West Public Health Observatory, Bristol, UK and Department of Social Medicine, University of

Bristol, Bristol, UK

Mark Kirkham

South West Public Health Observatory, Bristol, UK

Correspondence to: Dr M E Shaw, Department of Social Medicine, University of Bristol, Canynge Hall, Whiteladies Road, Bristol BS8 2PR, UK; Mary.shaw@bristol.ac.uk

Funding: Mary Shaw is funded by the South West Public Health Observatory. 